\title{
Chemical Reactors Catalyst bed and Analytical Solution of Complicated Partial Nonlinear Differential Equations by new Approach AKLM
}

\author{
MR Akbari ${ }^{1 *}$, Sara Akbari², Esmaeil Kalantari \\ ${ }^{1}$ Department of Civil Engineering and Chemical Engineering, Faculty of Chemical Engineering, University of Tehran, Iran. \\ 1,2Department of Chemical Engineering, Faculty of Chemical Engineering, Islamic Azad University, Iran.
}

*Corresponding author: MR Akbari, Department of Civil Engineering and Chemical Engineering, Faculty of Chemical Engineering, University of Tehran, Tehran, Iran.
Received Date: November 19, 2020

Published Date: December 10, 2020

\begin{abstract}
In this paper, for the first time, we investigate and solve a complicated highly partial nonlinear differential equations of chemical reactions in the cylindrical and spherical coordinates on the catalyst bed for the design of chemical reactors. We challenge and prove the power of this method that it can easily analyze the most difficult nonlinear problems in a completely analytical way, which we named it AKLM (Akbari Kalantari Leila's Method). Certainly we know that the chemical reaction process on the solid catalysts is very complex, and the governing differential equations governing them are nonlinearity complex. In this paper we present a new analytical solution which can easily analyze all such problems and make a great evolution in the nonlinear industries in the reactors design in chemical engineering. Finally, this scientific approach can create a great phenomenon in the analytical solution of nonlinear problems in engineering sciences, especially in the chemical and mechanical engineering.
\end{abstract}

Keywords: New method; Akbari-Kalantari-Leila's Method (AKLM); Reactor Catalytic bed; Partial Nonlinear Differential Equation; Cylindrical, Spherical and Cartesian Coordinates

\section{Introduction}

In the study, our aims introduce of accuracy, capabilities and power for solving complex non-linear partial differential in the reaction chemical on the reactor's catalyst bed. AKLM method can be successfully applied in various engineering fields such as mechanics (solid and fluid), electronics, petroleum industry, designing chemical reactors [1,2], and also in applied sciences (physics), economics and so on. It is worth noting that these two methods are convergent at any form of differential equations, including any number of initial and boundary conditions. During the solution procedure, it is not required to convert or simplify the exponential, trigonometric and logarithmic terms, which enables the user to obtain a highly precise solution. Besides, the methodology behind these techniques are completely understandable, easy to use, and users with common knowledge of mathematics will be capable of solving the most complicated equations at low calculation cost. As all experts know most of engineering actual systems behavior in practical are nonlinear process and analytical scrutiny these nonlinear problems are difficult or sometimes impossible. Our purpose is to enhance the ability of solving the mentioned nonlinear differential equations at chemical engineering and similar issues with a simple and innovative approach which entitled "AkbariKalantari-Leila Method" or "AKLM". He's Amplitude Frequency Formulation method [3-5] which was first presented by Ji-Huan He gives convergent successive approximations of the exact solution and Homotopy perturbation technique HPM [6]. It is necessary to mention that the above methods do not have this ability to gain the 
solution of the presented problem in high precision and accuracy so nonlinear differential equations such as the presented problem in this case study should be solved by utilizing new approaches like AGM [7-13] that created by Mohammadreza Akbari(in 2014). In recent years, analytical methods in solving nonlinear differential equations have been presented and created by Mohammadreza Akbari, these methods are called AYM (Akbari Yasna's Method in April 2020)and ASM [14] (Akbari Sara's Method in August 2019) and AKLM (Akbari Kalantari Leila Method in August 2020).These example somehow can be considered as complicated cases to deal with for all of the existed analytical methods especially in the design reactor in chemical engineering, which means old methods cannot resolve them precisely or even solve them in a real domain.

\section{Mathematical formulation of the Problem}

We consider an isothermal reaction and also, we assume governing chemical reaction equation on the solid catalyst bed in the chemical reaction are complicated and according of mathematical equations as follows:

1. By assuming that on the catalyst bed the reaction chemical for component $\mathrm{A}$ is as follows:

$$
\begin{aligned}
& A+(\text { catalyst }) \rightarrow B \\
& r_{A}=\eta\left(\frac{\partial C}{\partial r}\right)^{p}+\mu \sqrt[q]{1+\exp \left(v C+\varepsilon C^{2}\right)}
\end{aligned}
$$

2. Partial differential equation of chemical reaction in polar coordinates as follows:

$$
\frac{\partial C}{\partial t}=\frac{\alpha}{r^{n}} \frac{\partial}{\partial r}\left(r^{n} \frac{\partial C}{\partial r}\right)+V \frac{\partial C}{\partial r}+r_{A} ; C=C(r, t)
$$

Here the parameters $\mathrm{C}$ is concentration material $\mathrm{A}$ and so parameters $\alpha, \varepsilon, \mathrm{m}, \mathrm{s}, \mathrm{u}, \mathrm{p}, \mathrm{q}$ are the physical constant values of the chemical process and the value $\mathrm{V}$ is materials reactant in the chemical reactor.

$$
\begin{aligned}
& n=0 \rightarrow \text { Slab } \\
& n=1 \rightarrow \text { Cylindrical } \\
& n=2 \rightarrow \text { Spherical }
\end{aligned}
$$

\section{For cylindrical coordinate with radii $R(n=1)$}

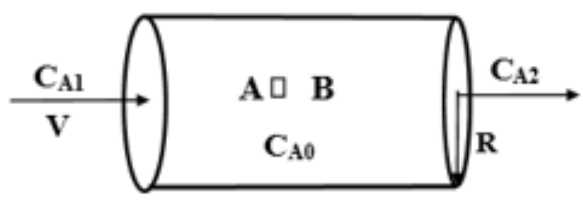

Figure1: The schematic system for cylindrical coordinate.

(Figure 1) By substituting the chemical reaction Equation (2) into the partial differential equation Equation (3), the result the following a complicated nonlinear partial differential equation in the cylindrical coordinate for reactants in catalytic chemical reactions as follows:

$$
\begin{gathered}
\frac{\partial C}{\partial t}=\frac{\alpha}{r} \frac{\partial}{\partial r}\left(r \frac{\partial C}{\partial r}\right)+V \frac{\partial C}{\partial r}+\eta\left(\frac{\partial C}{\partial r}\right)^{p}+ \\
\mu \sqrt[q]{1+\exp \left(v C+\varepsilon C^{2}\right)} \quad ; C=C(r, t)
\end{gathered}
$$

Boundary and initial condition are:

$$
\frac{\mathrm{d} C(0, t)}{\mathrm{d} r}=C_{A 1}, C(R, t)=C_{A 2}, C(r, 0)=C_{A \theta}
$$

physical values with metric units as follows:

$$
\begin{aligned}
& C_{A 1}:=0 ; C_{A 2}:=0.8 ; C_{A O}:=0.1 ; \eta:=0.2 ; \alpha:=0.8 \\
& V:=0.4 ; \varepsilon:=0.2 ; v:=0.2 ; \mu:=0.1 ; n:=1 ; R:=1 \\
& p:=3 ; q:=4
\end{aligned}
$$

Output of the solution process by new approach AKLM (AkbariKalantari-Leila Method) for partial nonlinear differential equation Equation (5), according to the boundary and initial Equation (6) and the physical values Equation (7) at the cylindrical coordinate system figure (1), the solution of the differential equation is obtained as follows:

$$
\begin{aligned}
C(r, t) & =\left(\frac{27}{250} r^{2}-\frac{1279100731}{3200000000} r^{4}+\frac{933500731}{3200000000}\right) \lambda^{3} \\
& +\left(\frac{881288157}{1600000000000}-\frac{881288157}{1600000000000} r^{4}\right) \lambda^{2} \\
& +\left(\frac{1101944297}{720000000000} r^{3}-\frac{1101944297}{720000000000}\right) \lambda \\
& -\frac{327249191}{2000000000} r^{2}+\frac{1927249191}{2000000000} ; \lambda:=\mathrm{e}^{-\frac{\alpha \pi^{2} n^{2} t}{R^{2}}}
\end{aligned}
$$

\section{Comparing the achieved solutions by Numerical Method and AKLM}

Due to the obtained solution from Equation (8) by AKLM, and Numerical method (Runge-Kutte 4th), we have the following comparisons:

(Figure 2) Concentration-time and reaction rate graphs as follows:

(Figure 3) In the concentration-time diagram for contour plot as follows:

(Figure 3) Charts of contour of AKLM solution for C(r,t). 

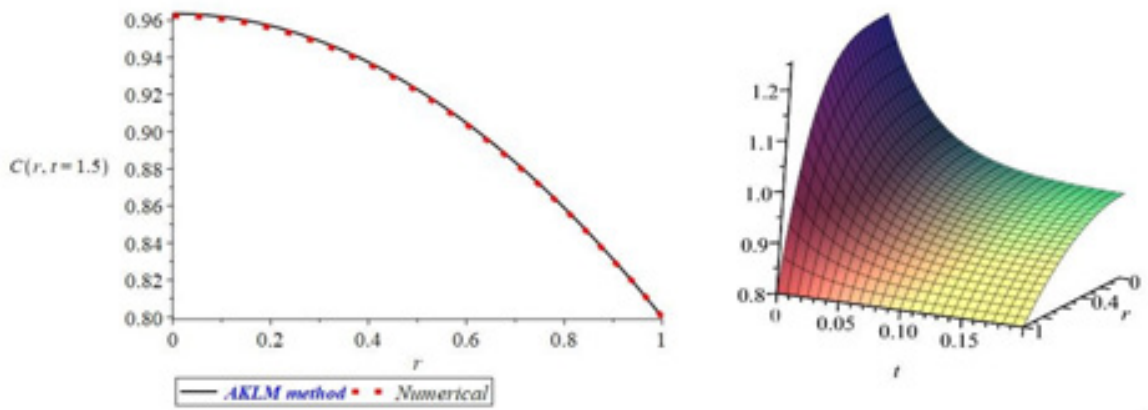

Figure 2: A comparison between AKLM and Numerical solution.
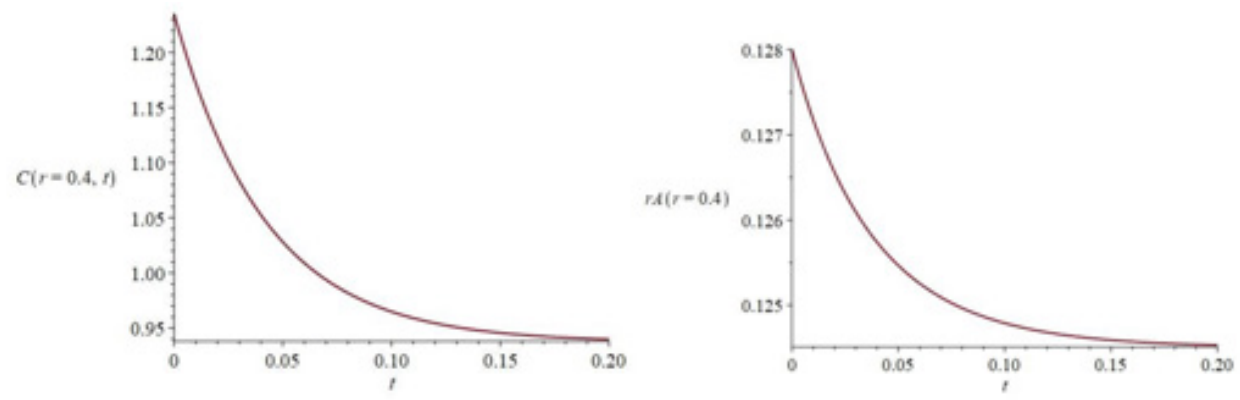

Figure 3: Concentration-time graph for radius location $r=0.4$ meter of achieved solutions by AKLM and for Concentration and also reaction rate.

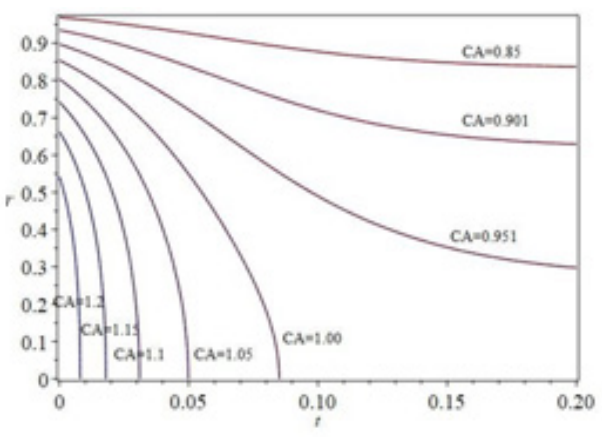

Figure 4: Charts of contour of AKLM solution for $\mathrm{C}(\mathrm{r}, \mathrm{t})$.

In the concentration-time diagram for contour plot as follows (Figure 4)

\section{For cylindrical coordinate $(n=2)$}

\section{$A+($ catalyst $) \rightarrow B$}

(Figure 5) As previous we assume that on the catalyst bed the reaction chemical for component $\mathrm{A}$ is as follows:

$$
r_{A}=\eta\left(\frac{\partial C(r, t)}{\partial r}\right)^{p}+\mu \sqrt[q]{1+\exp \left(v C(r, t)+\varepsilon C(r, t)^{2}\right)}
$$

(10) Partial differential equation of chemical reaction in spherical coordinates as follows:

$$
\frac{\partial C(r, t)}{\partial t}=\frac{\alpha}{r^{2}} \frac{\partial}{\partial r}\left(r^{2} \frac{\partial C(r, t)}{\partial r}\right)+V \frac{\partial C(r, t)}{\partial r}+r_{A}
$$

By substituting the chemical reaction Equation (10) into the partial differential equation Equation(11), the result the following a complicated nonlinear partial differential equation in the spherical coordinate for reactants in catalytic chemical reactions as follows:

$$
\begin{gathered}
\frac{\partial C}{\partial t}=\frac{\alpha}{r^{2}} \frac{\partial}{\partial r}\left(r^{2} \frac{\partial C}{\partial r}\right)+V \frac{\partial C}{\partial r}+\eta\left(\frac{\partial C}{\partial r}\right)^{p}+ \\
\mu \sqrt[q]{1+\exp \left(v C+\varepsilon C^{2}\right)} ; C=C(r, t)
\end{gathered}
$$

(12) Boundary and initial condition are: 


$$
\frac{\mathrm{d} C(0, t)}{\mathrm{d} r}=C_{A 1}, C(R, t)=C_{A 2}, C(r, 0)=C_{A D}
$$

previous the physical values are with metric units as follows:

$$
\begin{aligned}
C_{A 1} & :=0 ; C_{A 2}:=0.8 ; C_{A 0}:=0.1 ; \eta:=0.2 ; \alpha:=0.8 \\
V & :=0.4 ; \varepsilon:=0.2 ; v:=0.2 ; \mu:=0.1 ; n:=1 ; R:=1 \\
p & :=3 ; q:=4
\end{aligned}
$$

(14) Output of the solution process by new approach AKLM (Akbari-Kalantari-Leila Method) for partial nonlinear differential equation Eq.(12), according to the boundary and initial conditions Eq.(13) and the physical values Eq.(14) at the spherical coordinate system fig.(5), the solution of the differential equation is obtained as follows:

$$
\begin{aligned}
C(r, t) & =\left(\frac{991100731}{4000000000}-\frac{1279100731}{4000000000} r^{4}+\frac{9}{125} r^{2}\right) \lambda^{3} \\
& +\left(\frac{881288157}{2000000000000}-\frac{881288157}{2000000000000} r^{4}\right) \lambda^{2} \\
& +\left(\frac{1101944297}{960000000000} r^{3}-\frac{1101944297}{960000000000}\right) \lambda \\
& -\frac{327249191}{3000000000} r^{2}+\frac{2727249191}{300000000} ; \lambda:=\mathrm{e}
\end{aligned}
$$

Comparing the achieved solutions by Numerical Method and AKLM

Due to the obtained solution from Equation (15) by AKLM, and Numerical method (Runge-Kutte $4^{\text {th }}$ ), we have the following comparisons:

(Figure 6) Concentration-time and reaction rate graphs as follows (Figure 7)

In the concentration-time diagram for contour plot as follows (Figure 8)

\section{For cartesian coordinate $(j=0)$}

(Figure 9) As previous we assume that on the catalyst bed the reaction chemical for component $\mathrm{A}$ is as follows:

$$
\begin{gathered}
A+(\text { catalys } t) \rightarrow B \\
r_{A}=\eta\left(\frac{\partial C(x, t)}{\partial x}\right)^{p}+\mu \sqrt[q]{1+\exp \left(v C(x, t)+\varepsilon C(x, t)^{2}\right)}
\end{gathered}
$$

Partial differential equation of chemical reaction in cartesian coordinates as follows:

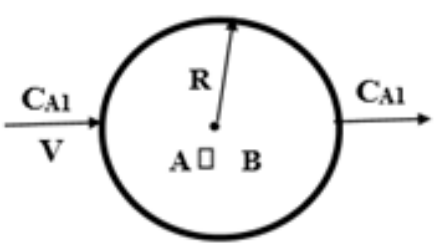

Figure 5: The schematic system for spherical coordinate.
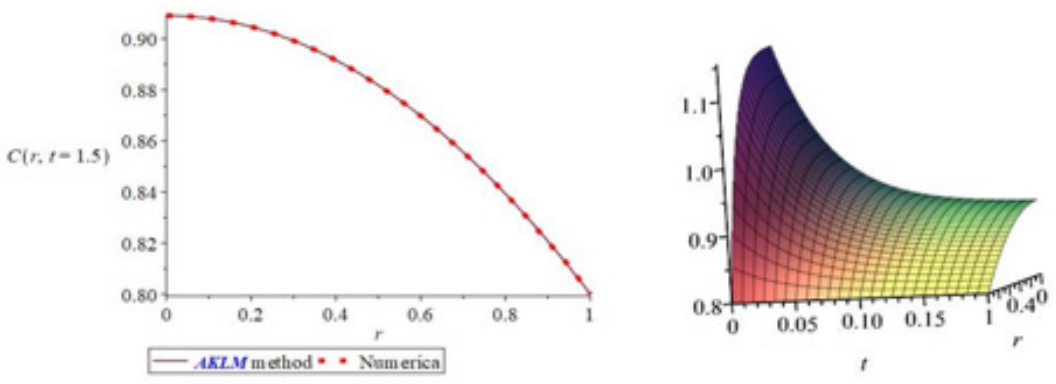

Figure 6: A comparison between AKLM and Numerical solution.
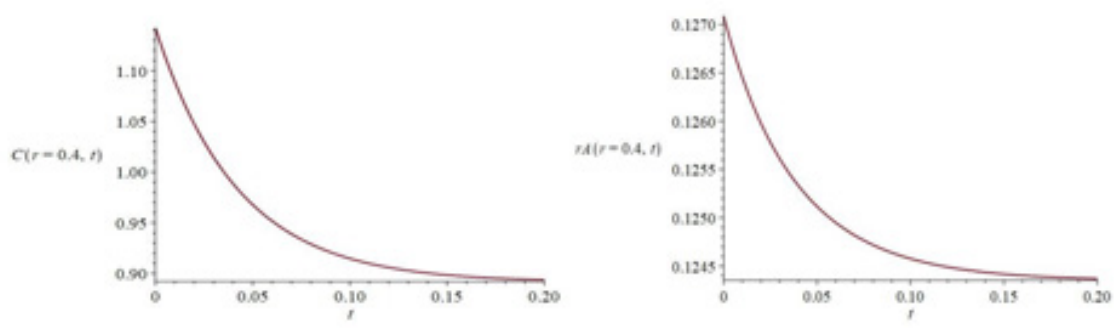

Figure 7: Concentration-time graph for radius location $r=0.4$ meter of achieved solutions by AKLM and for Concentration and also reaction rate. 


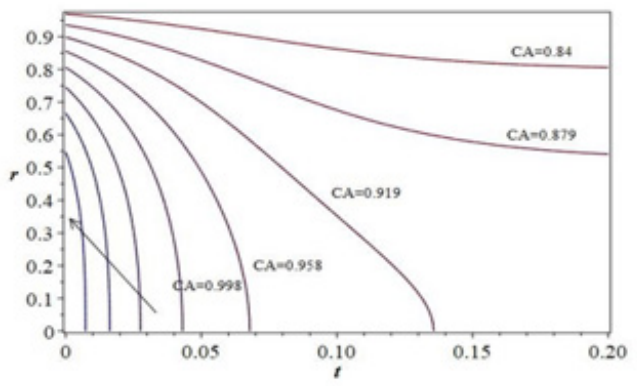

Figure 8: Charts of contour of ALKM solution for C (r.t).

$$
\frac{\partial C(x, t)}{\partial t}=\alpha \frac{\partial^{2} C(x, t)}{\partial x^{2}}+V \frac{\partial C(x, t)}{\partial x}+r_{A}
$$

By substituting the chemical reaction Equation (17) into the partial differential equation Equation (18), the result the following a complicated nonlinear partial differential equation in the cartesian coordinate for reactants in catalytic chemical reactions as follows:

$$
\begin{gathered}
\frac{\partial C}{\partial t}=\alpha \frac{\partial^{2} C}{\partial x^{2}}+V \frac{\partial C}{\partial x}+\eta\left(\frac{\partial C}{\partial x}\right)^{p}+ \\
\mu \sqrt[q]{1+\exp \left(v C+\varepsilon C^{2}\right)} ; C=C(x, t)
\end{gathered}
$$

Boundary and initial condition are:

$$
\frac{\mathrm{d} C(\mathbf{0}, t)}{\mathrm{d} x}=C_{A 1}, C(L, t)=C_{A 2}, C(x, 0)=C_{A \theta}
$$

As previous the physical values are with metric units as follows:

$$
\begin{aligned}
& C_{A 1}:=0 ; C_{A 2}:=0.8 ; C_{A 0}:=0.1 ; \eta:=0.2 ; \alpha:=0.8 \\
& V:=0.4 ; \varepsilon:=0.2 ; v:=0.2 ; \mu:=0.1 ; n:=1 \\
& L:=1 ; p:=3 ; q:=4
\end{aligned}
$$

Output of the solution process by new approach AKLM (AkbariKalantari-Leila Method) for partial nonlinear differential equation
Eq.(19), according to the boundary and initial conditions Equation (20) and the physical values Equation (21) at the slab coordinate system figure (9), the solution of the differential equation is obtained as follows:

$$
\begin{aligned}
C(x, t) & =\left(\frac{760700731}{2400000000}-\frac{1279100731}{2400000000} x^{4}+\frac{27}{125} x^{2}\right) \lambda^{3} \\
& +\left(\frac{293762719}{400000000000}-\frac{293762719}{400000000000} x^{4}\right) \lambda^{2} \\
& +\left(\frac{1101944297}{480000000000} x^{3}-\frac{1101944297}{48000000000}\right) \lambda \\
& -\frac{327249191}{1000000000} x^{2}+\frac{1127249191}{1000000000} ; \lambda=\mathrm{e}^{-\frac{\alpha \pi^{2} n^{2} t}{L^{2}}}
\end{aligned}
$$

Comparing the achieved solutions by Numerical Method and AKLM

Due to the obtained solution from Equation (22) by AKLM, and Numerical method (Runge-Kutte 4th), we have the following comparisons:

(Figure 10) Concentration-time and reaction rate graphs as follows:

(Figure 11) In the concentration-time diagram for contour plot as follows:

(Figure 12) Comparison of concentration graphs for cylindrical, spherical and Cartesian coordinates (Figure 13, 14)

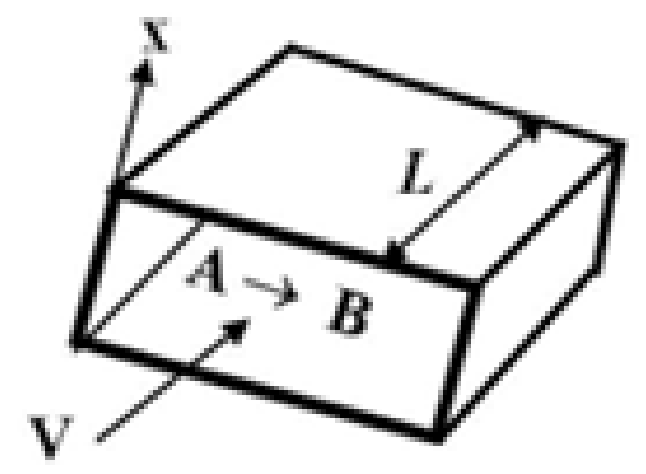

Figure 9: The Scematic system for slab coordinate 

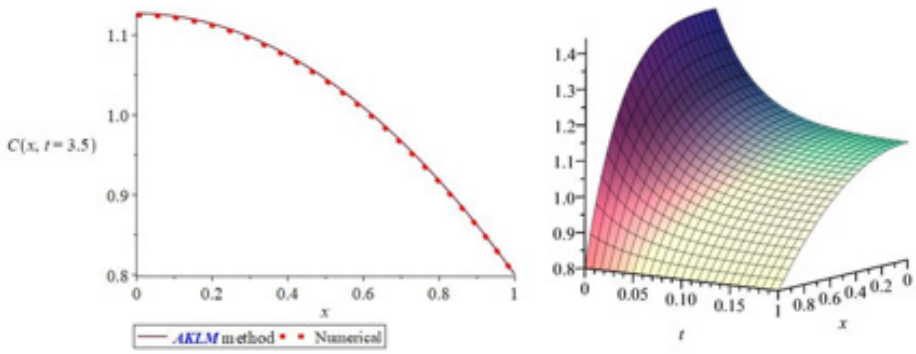

Figure 10: A comparison between AKLM and Numerical solution.
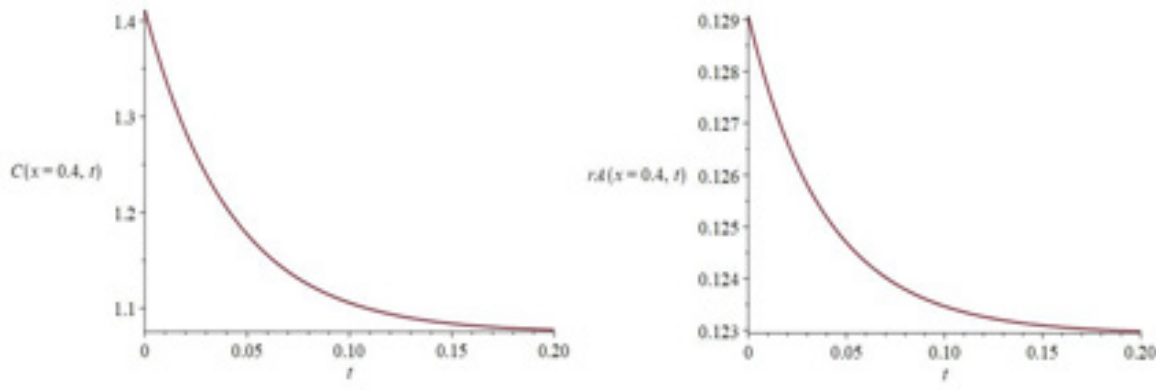

Figure 11: Concentration-time graph for radius location $x=0.4$ meter of achieved solutions by AKLM and for Concentration and also reaction rate.

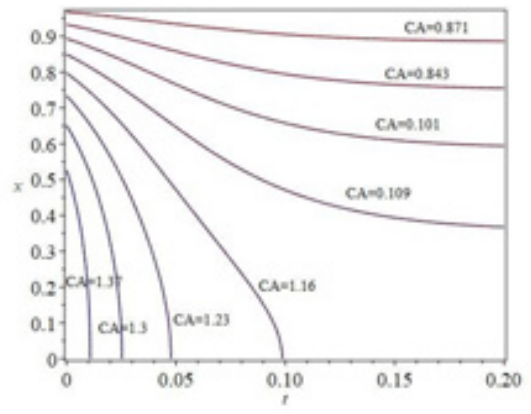

Figure 12: Charts of contour of AKLM solution for $C(x, t)$.

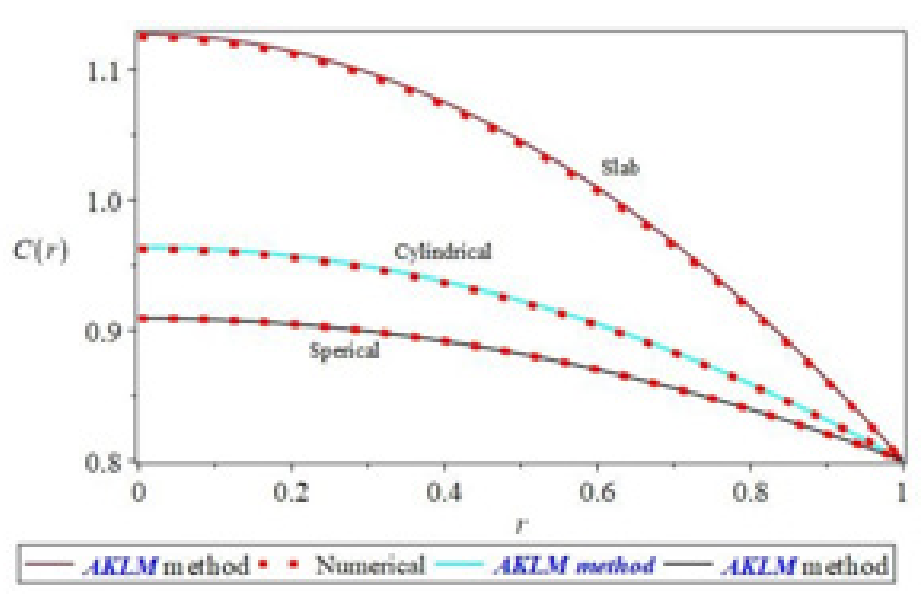

Figure 13: Comparison of concentration graphs for cylindrical, spherical and Cartesian coordinates for C(r). 


\section{Conclusion}

In this article, we proved that we can with new method (AKLM) all kinds of complicated practical problems related to nonlinear partial differential equations in the design of chemical reactors can be easily solved analytically. Obviously, most of the phenomena of chemical reactions in chemical reactors are nonlinear, so it is quite difficult to study and analyze nonlinear mathematical equations in this area, also we wanted to demonstrate the strength, capability and flexibility of the new AKLM method(Akbari-Kalantari-Leila Method). This method is newly created, and it can have high power in analytical solution of all kinds of industrial and practical problems in engineering fields and basic sciences for complex partial nonlinear differential equations.

\section{Acknowledgement}

None.

\section{Conflicts of Interest}

No Conflicts of Interest.

\section{References}

1. Bruce A Finlayson, Brigette M, Rosendall, University of Washington Seattle, WA 981951750, Bechtel Technology and Consulting San Francisco, CA 94119-3965.

2. D Zwillinger, Handbook of Differential Equations (New York: Academic Press, 1992).

3. JH He (2008) An improved amplitude-frequency formulation for nonlinear oscillators. International Journal of Nonlinear Sciences and Numerical Simulation 9 (2): 211.

4. ZF Ren, GQ Liu, YX Kang, HY Fan, HM Li, et al. (2009) Application of he's amplitude frequency formulation to nonlinear oscillators with discontinuities. Physica Scripta 80(4): 45003,
5. JH He (1998) Approximate analytical solution of Blasiu's equation. Commun Nonlin Sci Numer Simul 3(4): 260-263.

6. JH He (1999) Homotopy perturbation technique. J Comput. Methods Appl Mech Engrg. 178(3-4): 257-262.

7. Book Nonlinear Dynamic in Engineering by Akbari-Ganji's Method

8. MR Akbari, Sara Akbari, Esmaeil Kalantari (2020) Akbari-Ganji"s method "AGM" to chemical reactor design for non-isothermal and nonadiabatic of mixed flow reactors. Journal of Chemical Engineering and Materials Science 11(1): 1-9.

9. MR Akbari, DD Ganji, M Nimafar (2014) Significant progress in solution of nonlinear equations at displacement of structure and heat transfer extended surface by new AGM approach. Frontiers of Mechanical Engineering Journal 9: 390-401

10. AK Rostami, MR Akbari, DD Ganji, S Heydari (2014) Investigating JefferyHamel flow with high magnetic field and nanoparticle by HPM and AGM. Cent Eur J Eng 4(4): 357-370.

11. MR Akbari, DD Ganji, A Majidian, AR (2014) Ahmadi Solving nonlinear differential equations of Vanderpol, Rayleigh and Duffing by AGM. Frontiers of Mechanical Engineering 9: 177-190.

12. DD Ganji, MR Akbari, AR Goltabar (2014) Dynamic Vibration Analysis for Non-linear Partial Differential Equation of the Beam - columns with Shear Deformation and Rotary Inertia by AGM. Development and Applications of Oceanic Engineering (DAOE) 2325-3762.

13. MR Akbari, DD Ganji, AR Ahmadi, Sayyid H, Hashemi kachapi (2014) Analyzing the Nonlinear Vibrational wave differential equation for the simplified model of Tower Cranes by (AGM). Frontiers of Mechanical Engineering 9(1): 58-70.

14. MR Akbari, M Nimafar, DD Ganji, MM Akbarzade (2014) Scrutiny of nonlinear differential equations Euler Bernoulli beam with large rotational deviation by AGM. Springer 9:402-408.

15. MR Akbari, Sara Akbari, Esmaeil Kalantari (2020) A Study about Exothermic Chemical Reactor by ASM Approach Strategy. Crimson Publishers Wings to the Research. 